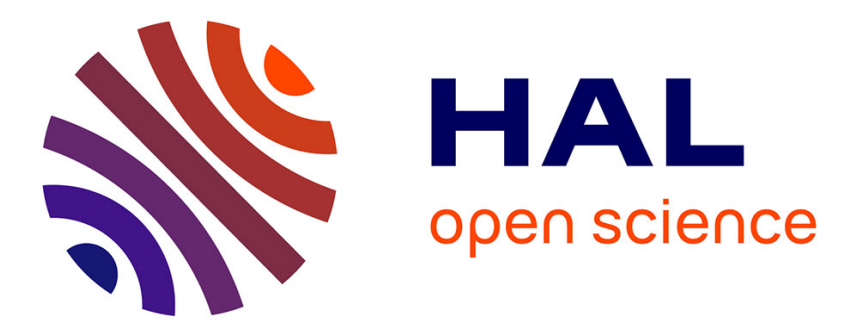

\title{
Localisation et cinétiques de deux antibiotiques aminoglycosidiques à potentialité toxique différente. La gentamicine et l'amikacine dans les cellules sensorielles de l'oreille interne
}

\author{
C. Chappert, C. Aurousseau, J.-P. Erre, D. Dulon, J.-M. Aran
}

\section{To cite this version:}

C. Chappert, C. Aurousseau, J.-P. Erre, D. Dulon, J.-M. Aran. Localisation et cinétiques de deux antibiotiques aminoglycosidiques à potentialité toxique différente. La gentamicine et l'amikacine dans les cellules sensorielles de l'oreille interne. Journal de Physique IV Proceedings, 1994, 04 (C5), pp.C5431-C5-432. 10.1051/jp4:1994590 . jpa-00253085

\section{HAL Id: jpa-00253085 https://hal.science/jpa-00253085}

Submitted on 1 Jan 1994

HAL is a multi-disciplinary open access archive for the deposit and dissemination of scientific research documents, whether they are published or not. The documents may come from teaching and research institutions in France or abroad, or from public or private research centers.
L'archive ouverte pluridisciplinaire HAL, est destinée au dépôt et à la diffusion de documents scientifiques de niveau recherche, publiés ou non, émanant des établissements d'enseignement et de recherche français ou étrangers, des laboratoires publics ou privés. 


\title{
Localisation et cinétiques de deux antibiotiques aminoglycosidiques à potentialité toxique différente. La gentamicine et l'amikacine dans les cellules sensorielles de l'oreille interne
}

\author{
C. CHAPPERT, C. AUROUSSEAU, J.-P. ERRE, D. DULON et J.-M. ARAN
}

INSERM U 229, Audiologie Expérimentale, Laboratoire Audiologique Expérimental, Hôpital Pellegrin, 33076 Bordeaux, France

\begin{abstract}
In order to determine the relation between uptake of aminoglycoside antibiotic (AA) molecules by sensory cells of the inner ear and their ototoxicity, we studied the kinetics in cochlear and in vestibular cells of two AAs: gentamicin (GM)(both cochleo- and vestibulo-toxic) and amikacin (AK)(mainly cochleo-toxic). Guinea pigs were treated during six days with one daily injection of GM $(60 \mathrm{mg} / \mathrm{kg} /$ day) or AK (450 mg/kg/day). AAs were detected in isolated cells using immunocytochemical technique with scanning laser confocal microscopy. Results demonstrate a rapid uptake and a very slow clearance of both AAs from cochlear and vestibular hair cells. Particularly GM and AK are detected in type I and type II hair cells of the utricles and cristae ampullaris. The presence of these two molecules with different toxic potentialities towards the cochlea or the vestibule in cochlear as well as in vestibular hair cells indicate that the selective toxicity of aminoglycosides cannot be explained solely on the basis of particular uptake and accumulation in the different sensorineural receptors.
\end{abstract}

Les antibiotiques aminoglycosidiques sont bien connus en thérapeutique clinique comme des molécules pouvant entraîner des pathologies envers les structures sensorielles de l'oreille interne : la cochlée (organe de l'audition) et le vestibule (organe de l'équilibre). La gentamicine et l'amikacine sont deux antibiotiques aminoglycosidiques aux spécificités tissulaires différentes: la gentamicine est un antibiotique affectant à la fois les cellules cochléaires et vestibulaires alors que l'amikacine est préférentiellement cochléotoxique (Aran 1982).

La pharmacocinétique des aminosides dans les fluides labyrinthiques (qui représentent la voie d'accès obligatoire aux structures neurosensorielles non vascularisées de l'oreille interne), et dans les tissus cochléaires et vestibulaires indique que les antibiotiques aminoglycosidiques cochléo- ou vestibulo-toxiques, comme l'amikacine et la gentamicine ont des cinétiques identiques (Dulon et coll. 1986). Cela n'explique donc pas la toxicité sélective de ces deux antibiotiques. Cependant la corrélation de la distribution cochléaire de la gentamicine en un gradient baso-apical et radial avec les altérations des cellules sensorielles cochléaires correspondantes a laissé supposer que les mécanismes de toxicité 
spécifique seraient liés à ceux de la pénétration de l'antibiotique aminoglycosidique dans les cellules (Aran, 1990, Hiel et coll. 1992, 1993a, 1993b).

La cinétique de capture et d'élimination de la gentamicine et de l'amikacine déterminée par immunofluorescence et microscopie confocale à balayage laser, a donc été comparée au niveau des cellules ciliées de la cochlée et des récepteurs vestibulaires chez le cobaye. La gentamicine et l'amikacine sont administrées par injections sous-cutanées pendant 6 jours à des doses respectives de $60 \mathrm{mg} / \mathrm{kg} / \mathrm{j}$ et $450 \mathrm{mg} / \mathrm{kg} / \mathrm{j}$ (équivalentes à 20 et 30 fois la dose thérapeutique humaine).

La gentamicine et l'amikacine sont localisées de manière similaire, sous la plaque cuticulaire des cellules ciliées externes (CCE) de la cochlée. La capture de la gentamicine par les CCEs est rapide et précède l'apparition des modifications de la fonction auditive. Ceci est également observé avec l'amikacine. L'élimination de la gentamicine se fait en deux étapes : une étape rapide et une autre beaucoup plus lente (Hiel et coll. 1992a, 1993, Dulon et coll. 1993). A la dose d'amikacine administrée ici, il ne semble par contre pas y avoir de phénomène d'élimination rapide étant donné que 30 jours après la dernière injection, l'amikacine est présente dans les cellules ciliées externes à un niveau identique au pic atteint lors de la capture. II pourrait y avoir là une explication à la forte cochléotoxicité de l'amikacine. Par ailleurs, la gentamicine et l'amikacine sont toutes les deux également détectées dans les cellules de type I et II de l'utricule et des crêtes ampullaires.

La présence de ces deux molécules, à potentialité toxique différente envers la cochlée et le vestibule, aussi bien dans les cellules cochléaires que vestibulaires indique que la toxicité sélective des aminoglycosides ne peut s'expliquer uniquement par une capture et d'une accumulation particulière dans les différents récepteurs sensoriels (Dulon et col. 1986).

\section{REFERENCES}

Aran JM. (1982) Cibles cochléaires et vestibulaires des aminoglycosides. In : Néphrotoxicité, Ototoxicité médicamenteuses. Fillastre (ed.), Paris, Inserm, 377-386.

Aran JM (1990). Physiopathology of sensory hair cells. In vivo and in vitro studies aminoglycoside uptake and toxicity. In : Grandori, F., Cianfrone, G., Kemp D.T. (eds). Cochlear mechanisms and otoacoustic emissions, Bâle. Adv. Audiol, Karger, 7, 42-46.

Dulon, D., Aran JM, Zajic CG, Schacht J (1986) Comparative uptake of gentamicin netilmicin and amikacin in the guinea pig cochlea and vestibule. Antimicrob. Agents Chemoth., 30, 96-100.

Dulon D, Hiel H, Aurousseau C, Erre JP, Aran JM (1993) Pharmacokinetics of gentamicin in the sensory hair cells of the organ of Corti : rapid uptake and long-term persistence. CR Acad Sci Paris, Sciences de la Vie, 316, 682-687

Dulon D, Zajic CG, Aran JM, Schacht J (1989): Aminoglycosides antibiotics impair calcium entry but not viability and motility in isolated cochlear outer hair cells. J. Neurosci. Res. 24:338-346.

Hiel H, Erre JP, Aurousseau, C, Bouali, R, Dulon D, Aran JM (1993). Gentamicin uptake by cochlear hair cells procedes hearing impairement during chronic treatment. Audiology 32, 78-87

Hiel H, Bennani H, Erre JP, Aurousseau A, Aran JM (1992a) Kinetics of gentamicin in cochlear hair cells after chronic treatment. Acta Otolaryngol (Stockhoim) 112, 272-277

Hief H, Schamel A, Erre JP, Hayashida T, Dulon D, Aran JM (1992). Cellular and subcellular localization of tritiated gentamicin in the guinea pig cochlea following combined treatment with ethacrynic acid. Hearing Res., 57, 157-165. 